\title{
Towards a Blockchain-based Identity and Trust Management Framework for the IoV Ecosystem
}

\author{
Anastasia Theodouli, Konstantinos Moschou \\ Konstantinos Votis, Dimitrios Tzovaras \\ CERTH / ITI \\ Thessaloniki, Greece \\ anastath,konsmosc,kvotis, tzovaras@iti.gr
}

\author{
Jan Lauinger, Sebastian Steinhorst \\ Embedded Systems and Internet of Things \\ Technical University of Munich, TUM \\ Munich, Germany \\ jan.lauinger, sebastian.steinhorst@tum.de
}

\begin{abstract}
The past decade has seen a huge growth not only in the Internet of Things (IoT) but also in the Internet of Vehicles (IoV) from both academia and industry. Autonomous Vehicles (AVs) combine a variety of sensors, IoT devices, control units, gateways, etc. Therefore, the software of the different sensors and IoT devices needs to be updated to the latest version by the Software Vendors. This paper proposes a blockchain-based identity and trust management framework for the IoV ecosystem, that aims to provide secure software updates. The proposed framework consists of two processes: the identification and registration of the entities of the ecosystem, and the authentication of the entities which relies on established W3C standards for defining verifiable credential presentation, verification, and revocation. We illustrate the feasibility of our approach in a case study.
\end{abstract}

Keywords-blockchain; Self-Sovereign Identity(SSI); Decentralized Identifiers(DIDs); Verifiable Credentials(VC); software update; Autonomous Vehicles; identity management; Internet of Things(IoT); Internet of Vehicles(IoV)

\section{INTRODUCTION \& MOTIVATION}

IoV ecosystem allows the establishment of a communication channel among vehicles so that they can exchange information. This information exchange has numerous benefits such as increasing road safety, and better traffic control. However, in the IoV ecosystems, there are several vulnerabilities that can be exploited which can have detrimental effects such as provoking accidents. As such, it is necessary to ensure the authenticity of the data exchanged in the established communication channel. To this end, we present our first contribution, which is an Identity and Trust Management Framework that allows the identification and authentication of the stakeholders of the IoV ecosystem and secures their communication. The framework leverages the Blockchain technology to save the Identifiers of the stakeholders in a decentralised way which eliminates the single point of failure while increasing availability and at the same time it overcomes the necessity of having a trusted third party, centralised Certificate Authority (CA) for managing the identifiers. The centralised approach is more prone to the single point of failure and it is allows for easier data breaches in case that the server hosting the certificates is compromised. It's worth noting that the proposed framework follows the proposed W3C standards for decentralised identifiers [1]. Furthermore, it uses Verifiable Credentials so as to build Trust among the stakeholders of the IoV ecosystem. Credentials are sets of claims (or statements), made by one entity, i.e. the Issuer, (normally) for another entity, i.e. the Holder (or Prover). Verifiable Credentials wrap the claims mentioned above with a set of metadata and digital proofs (digital signature is the most usual type of digital proof) which cryptographically prove who is the Issuer of the Credentials [2]. Vehicles are equipped with sensors, control units, and IoT devices; the maintenance of these devices must be done by authorised services over a secure communication channel. To this end, we present our second contribution which is the application of the Identity and Trust Management Framework mentioned above to an important use case in the IoT domain, i.e. the software update of the IoT devices software embedded in the vehicle, in a secure and private way.

The remainder of this paper is structured as follows. In Section II, we present background on blockchain technology and similar works. In Section III, we present our proposed Framework, by outlining, (i) the blockchain platform that will be used after assessing its suitability for our IoV ecosystem necessities and comparing it with other candidate blockchain platforms, (ii) its stakeholders and their roles, and (iii) its processes. Finally, in Section IV, we conclude the paper and define the next steps for this work.

\section{BACKGROUND \& RELATED WORK}

\section{A. Blockchain Technology}

In blockchain technology, cryptography and, essentially, hash functions bind data blocks together and enable data integrity checks. Consensus protocols, timely consecutive cryptography, and a decentralized network design extend the data linking concept to establish the attractive guarantees. Ledger data immutability (tamper resistance), integrity, traceability, transparency, and availability count to the guarantees that enable secure and reliable bookkeeping of data. 
One of the branches that originated from blockchain research is the consortium-based Distributed Ledger Technology (DLT) [3]. Compared to public blockchains, the most distinctive characteristic of consortium-based DLT is that only authorized nodes participate in the network [4]. This restriction enables the network to utilize voting based consensus protocols such as Practical Byzantine Fault Tolerance (PBFT) which increase scalability of the network. As a result, consortium blockchains achieve faster transaction finality and low-delay verification times of transactions.

Identity Management (IdM) in the IoV represents one of the candidates that require the benefits of DLT [5]. Moreover, the IoV with multiple stakeholders as infrastructure participants requires a consortium-based DLT approach. Reasons therefore are the vehicle insurance and the legal requirement of technical inspection of vehicles from trusted associations to receive, for instance, the car license and number plate. Such processes rely on multiple verified authorities that contribute to the admission of a vehicle. This means that the ledger between the authorities depends on input from multiple authorized sources. External input in form of transactions from authorized participants should pass a fair consortium decision to lower trust boundaries between consortium partners [6]. Afterwards, the tamperproof bookkeeping property of DLT excludes the third party that manages bookkeeping between stakeholders.

External input verification among closed environment DLT frameworks is state-of-the-art research and requires explicit examination during application design [7]. Further, the focus on privacy preserving techniques [8], scalability solutions [9], and blockchain types (public, private and consortium blockchain networks) [10] drive distributed ledger framework development to tailored design choices. Different DLT architectures address the aforementioned problems differently and introduce different pros and cons. Hence, the right selection of a DLT framework is important with the goal to design IdM. In our case, IoV-specific requirements as well as the selection between independent, federated, or Self-Sovereign Identity (SSI) [5] IdM approaches dominate our framework selection in section III-A.

\section{B. Related Work}

In this section, we mention existing works that focus on (i) addressing security in software/firmware updates on Internet of Things(IoT) (ii) IdM using the Blockchain technology.

In [11], the authors introduced a consortium blockchainbased solution among the AVs manufacturers using smart contracts to ensure authenticity and integrity firmware updates tailored for AVs. AVs are participating in the distribution process of the firmware updates for high availability and fast delivery compared to centralized third party services.

In [12], Lee and Lee et al. proposed a blockchain-based solution to ensure the integrity of firmware updates on embedded IoT devices instead of depending on a centralized vendor network. For the distribution of updates, a peer-topeer file sharing network such as BitTorrent is proposed to ensure integrity and versions tractability of updates.

In [13], Yohan and Lo et al. proposed a blockchain-based framework that aims to provide secure verification on the firmware released by the device manufacturer. The proposed framework uses smart contracts and is designed based on the architecture of Ethereum[14].

In Ockam network [15], a decentralized blockchain IdM platform that enables interoperability, security, privacy, trust and reliability among IoT devices is developed. It is implemented with open standards developed by the World Wide Web Consortium (W3C) and the Decentralized Identity Foundation (DIF) for decentralized identifiers (DIDs) and verifiable credentials to ensure interoperability of identities. The developed platform consists of processes such as registration, authentication and authorization between entities of the IoT ecosystem.

As regards the works mentioned above we differ in that (i) we focus on the IoV ecosystem, (ii) we require/combine identity management as a necessity for our secure software update use case, (iii) we also address the issue of revocation of the credentials which is important in advanced identity management solutions, and (iv) we map the stakeholders of our framework to the standardization registration paradigms of the European Union (EU).

\section{Proposed Identity \& Trust Management FRAMEWORK}

The proposed identity and trust management framework for secure communication proceeds a comparison of DLT frameworks and an identification procedure of IoV stakeholders. The following sections explain this process by introducing the evaluation criteria for the selection of a DLT framework in the first place. The following comparison of DLT frameworks determines Indy as the most suitable solution for the IoV use case. The section about infrastructure stakeholders identifies and maps stakeholders to identity roles of Indy. This approach represents the prerequisite to determine the logical process of the IdM use case for secure communication.

\section{A. Blockchain Platforms Evaluation}

The following bullet points summarize the evaluation criteria for the selection of a DLT framework for IdM.

- Existing identity management concepts

- Missing identity management concepts that need to be developed

- Existence of privacy preserving techniques

- Framework architecture extendibility

As stated in section II-A, the IoV scenario requires a consortium-based DLT solution with scalability and realtime capabilities to handle location and velocity dynamics that occur in the IoV. 
Table I

DLT FRAMEWORK COMPARISON IN RESPECT OF IDENTITY MANAGEMENT IN THE IOV

\begin{tabular}{|l|l|l|l|}
\hline $\begin{array}{l}\text { DLT Frame- } \\
\text { work }\end{array}$ & IdM Support & Privacy & Expandability \\
\hline \hline Sawtooth & Medium & Low & High \\
\hline Fabric & $\begin{array}{l}\text { Medium } \\
\text { (Structure of } \\
\text { Authorities) }\end{array}$ & $\begin{array}{l}\text { Medium } \\
\text { (Channels) }\end{array}$ & High \\
\hline Indy & High & High & High (Plugins) \\
\hline
\end{tabular}

1) Framework Design: The dominating components of the Hyperledger Sawtooth framework are the transaction client and processor [16]. Together, they make up a transaction family which allows to modify the ledger state. Transaction clients compose and sign transactions which reach the transaction processor for verification. Validator nodes handle transaction processor assignments and submit successfully verified transactions to the Sawtooth journal component which is responsible for appending the blocks of transactions to the ledger.

In the Hyperledger Fabric framework, clients register at authorities of Fabric which enable clients to discover Fabric peers. Next, clients initiate signed transaction proposals, collect signed endorsements from peers, inspect responses, and finally broadcast transactions to an ordering service. The ordering service verifies signatures, orders transactions, creates blocks, and delivers blocks to ledger peers which append the blocks to the ledger. The concept of Fabric is scalable as it allows to connect multiple Fabric networks.

The Hyperledger Indy framework follows a similar transaction design compared to Sawtooth. The Indy client System Development Kit (SDK) can be seen as the transaction client that submits predefined transaction requests. Indy ledger transactions cover all important ledger interactions around Decentralized Identifier (DID) management. DID creation and querying, key rotation, credential schema creation, credential definition creation, etc belong to the Indy transaction repertoire [17]. Additionally, the Indy SDK supports local credential management with the help of wallets. Wallets store credentials, cryptographic key pairs and can be used for caching of Decentralized Identifiers (DIDs).

All frameworks rely on scalable voting based consensus algorithms such as PBFT that allow high transaction throughput [3].

2) Identity Management Suitability: The Sawtooth framework provides an identity transaction family. The identity transaction family implements client roles and permissions (policies) which allow authorization. Moreover it is possible to set up cross transaction family namespace restrictions. Missing features of IdM encompass maintenance and revocation design. Privacy enhancing approaches for verification of identities is left to the system designer.
Fabric channels enable clients to access specific chain information. Information separation/access is thereby possible which contributes to data privacy capabilities [18]. Apart from that, Fabric allows clients to prevent data exposure on the global blockchain storage. Instead, clients can utilize their own separated storage. Fabric allows authorization of clients at the initial authorities which provide access to Fabric peers.

Verifiable credential features of Indy enable to bind multiple private users to public credential schemata and authorities, which creates cryptographically sealed trust connections. With that, it is possible to implement trusted identity management procedures such as registration, authentication, authorization, and revocation between clients that do not store their DIDs in a publicly accessible ledger. Indy moreover supports transaction flows that cover legal nuances even though such flows remain optional. For instance, Transaction Author Agreement (TAA) bind explicit confirmation information to transactions. In cases of adding private information to the immutable ledger, such explicit agreements enhance opportunities from the technical perspective. For this purpose, Transaction Author Agreement Acceptance Mechanisms List (TAA AML) store acceptance mechanisms in the configuration ledger of the Indy nodes. Indy plugins support the creation of customized transactions and respective validations and resemble the transaction family design of Sawtooth. Missing IdM extension could be extended through plugins. For example, designing dynamic validations which let consortium members actively interact during transactions could provide consortium based verification of transaction data and, thereby, consortium-only control.

Indy represents the platform with most out of the box functionalities for the development of advanced IdM solutions. Table I summarizes the comparison and shows the suitability of Indy for advanced IdM which represents the result of the evaluation.

\section{B. Identified Stakeholders and Processes of the Framework}

In this section, we present the identified stakeholders of the system and their role.

As regards the registration of the vehicles to the system, we have followed the paradigm provided by the European Union [19] in order to identify the stakeholders involved. The list of the identified stakeholders appears in III-B below.

In Table II we mark with bold the stakeholders that are involved in the use case that we describe in Figure 1 below. Although we followed the European Union paradigm mentioned above to identify the stakeholders of our system in a concrete and realistic way, in the rest of this Section, we will elaborate only on the role of the stakeholders who participate in the Use Case presented in the Figure 1 below.

1) Role of the Trustee and the Steward: In Hyperledger Indy, Trustees and Stewards are specific roles given to users who play an important role in building the trust in the

DOI: $10.1109 /$ GIOTS49054.2020.9119623 
Table II

FRAMEWORK STAKEHOLDERS

\begin{tabular}{|l|l|l|}
\hline Stakeholder & $\begin{array}{l}\text { Hyperledger Indy } \\
\text { Role }\end{array}$ & Ecosystem Role \\
\hline \hline- & Trustee & Steward creator \\
\hline- & Steward & $\begin{array}{l}\text { Trust Anchors Cre- } \\
\text { ator }\end{array}$ \\
\hline Government & Trust Anchor & $\begin{array}{l}\text { Issuer of Credential } \\
\text { Schemas }\end{array}$ \\
\hline Vehicle manufacturer & Trust Anchor & $\begin{array}{l}\text { Issuer of Credential } \\
\text { Definition }\end{array}$ \\
\hline $\begin{array}{l}\text { Pre-market device soft- } \\
\text { ware vendor }\end{array}$ & Trust Anchor & $\begin{array}{l}\text { Issuer of Credential } \\
\text { Definition }\end{array}$ \\
\hline $\begin{array}{l}\text { Pre-market device soft- } \\
\text { ware vendor API }\end{array}$ & User & Prover/Verifier \\
\hline $\begin{array}{l}\text { Post-market device } \\
\text { software vendor }\end{array}$ & Trust Anchor & $\begin{array}{l}\text { Issuer of Credential } \\
\text { Definition }\end{array}$ \\
\hline $\begin{array}{l}\text { Post-market device } \\
\text { software vendor API }\end{array}$ & User & Prover/Verifier \\
\hline $\begin{array}{l}\text { Vehicle Owner or Trans- } \\
\text { portation Authority (Ve- } \\
\text { hicle Operator) }\end{array}$ & Trust Anchor & $\begin{array}{l}\text { Issuer of Credential } \\
\text { Definition }\end{array}$ \\
\hline $\begin{array}{l}\text { Vehicle Registration } \\
\text { Certificate Authority }\end{array}$ & Trust Anchor & $\begin{array}{l}\text { Issuer of Credential } \\
\text { Definition }\end{array}$ \\
\hline $\begin{array}{l}\text { Insurance Company } \\
\text { VAT payee / tax office }\end{array}$ & Trust Anchor & $\begin{array}{l}\text { Issuer of Credential } \\
\text { Definition }\end{array}$ \\
\hline $\begin{array}{l}\text { Roadworthiness Anchor } \\
\text { Provider Authority }\end{array}$ & $\begin{array}{l}\text { Issuer of Credential } \\
\text { Definition }\end{array}$ \\
\hline Vehicle & $\begin{array}{l}\text { Issuer of Credential } \\
\text { Definition }\end{array}$ \\
\hline
\end{tabular}

network [20]. However, in the paradigm that we follow [21] Trustees and Stewards are created programmatically within a set of pre-defined transactions, the so-called domain genesis transactions; the bootstrapping of the system by assigning/binding the roles of Trustees and Stewards to real users who can truly build trust to the system due to their inherent properties, is a matter of future work. Trustees create users assigned the Role of Stewards and Stewards create users assigned the role of Trust Anchors ${ }^{1}$; the latter, are entitled to write transactions to the Ledger, e.g. for publishing to the Ledger Decentralised Identifiers and for creating and publishing to the Ledger Credential Schemas and Credential Definitions. Note that each Steward is also a Trust Anchor.

In the following, we assume that a Trustee has created a user in the system assigned the role of Steward who is responsible for onboarding to the System, by assigning the Role of Trust Anchor to the Government, the Premarket device software vendors, and the post-market device software vendors. To do this, first, a secure communication is established between the Steward and each of the aforementioned stakeholders. This involves the creation of unique pairwise DIDs, one for the Steward and one for the each Stakeholder, respectively. Along with the DIDs an

\footnotetext{
${ }^{1}$ Note that the 'Trust Anchor' role has been renamed to 'Endorser'.
}

associated verification key and signing key are also created. These pairwise DIDs are private data stored locally within software applications which are controlled by the Steward and the Stakeholders, the so-called 'wallets'.

For the establishment of the secure communication, a mutual authentication process is done during which both parties should prove to each other that they hold the signing keys corresponding to the pairwise DIDs. After the successful completion of the mutual authentication, the messages exchanged are encrypted with the verification keys of the two parties to ensure the confidentiality of the exchanged messages. Then, the Government issues a DID and sends it to the Steward along with its verification key, the Steward publishes the DID and the verification key to the ledger and assigns to the Government the role of Trust Anchor. All data published to the Ledger are available to all users of the network who have at least read access; also, they are considered to be trusted by all the users of the network. The same procedure will occur for the pre-market and postmarket device software vendors. In the rest of this paper, we will refer to the process of (i) the secure connection establishment between a Trust Anchor and a new user, (ii) the subsequent write of the DID and verification key to the ledger by the Trust Anchor, and (iii) the assignment of a role to the new User as Registration of the user to the system. Furthermore, the process of creating unique DIDs (along with the corresponding verification and signing keys) is considered to be the process of Identification ${ }^{2}$. A list of the processes of the system is displayed in Table III below.

Table III

FrAMEWORK PROCESSES

\begin{tabular}{|l|l|}
\hline Process ID (PID) & Process Name \\
\hline \hline 1 & Identification and \\
& Registration \\
\hline 2 & Authentication (VC \\
& Presentation, VC \\
& Verification, VC \\
& Revocation) \\
\hline
\end{tabular}

2) Role of the Government: The Government should be first registered in the System by a Steward (or Trust Anchor) having the role of Trust Anchor. Then, it issues and publishes to the Ledger Credential Schemas which can be used as 'standards' for the Issuance of Credentials by the pre-market and post-market device software vendors.

3) Role of Pre-market and Post-market device software vendor: Pre-market and post-market device software vendors are the designers and manufacturers of devices such as video camera, lidar, radar, odometers, Global Positioning

\footnotetext{
${ }^{2}$ We consider this process as Identification of the user, since it encompasses the creation of the unique identifiers of the user. Note though, that in the generic case, one user can have multiple DIDs to avoid identity correlation.
} 
System (GPS), inertial measurement unit (IMUs), and embedded electronics like Electronic Control Units (ECUs) that are embedded to the vehicle before or after its transfer to an Owner or Operator, respectively. They are both responsible for the regular updates of the software that is installed within the devices.

The Pre-market and Post-market device software vendors issue Credential Definitions for the Pre-market and Postmarket device software vendor APIs, repsctively. These Credentials Definitions are based on the Credential Schemas that were published to the Ledger by the Government.

4) Role of Pre-market and Post-market device software vendor API: The Pre-market device software vendor APIs are APIs located at the Cloud that contain services and endpoints for the provision of the device software updates. The steps of the process for the authentication of the API by the Vehicle are the following:

1) The API first establishes a secure communication with the Vehicle.

2) The Vehicle sends a Proof Request for the Credentials regarding the kind of software the API is entitled to provide updates for.

3) The API retrieves the related Credentials from its wallet, and creates a Credential Presentation including a Proof that the Credentials were issued to them by the device software vendor and sends the Presentation to the Vehicle.

4) The Vehicle verifies through the Ledger the Proof included in the Credential Presentation regarding the Issuer of the Credentials, i.e the Software Vendor.

The process is displayed in Figure 1 below. The above process is only the half of the mutual authentication; the authentication is mutual, so the Vehicle will have also to present Credentials to the API that prove that the device to be updated is among the devices that have been manufactured by the Software Vendor who performs the update. The Issuer of these Credentials is the software Vendor. The API verifies through the Ledger the Proof included in the Credentials regarding the Issuer of the Credentials, i.e the Software Vendor. The Software Vendor is trusted by both entities since its DID and verification are published and resolvable from the Ledger by a Trusted Anchor.

After successfully completing the mutual authentication, the API should present to the Vehicle requested Credentials containing the digital fingerprint (e.g. a hash) of the Code corresponding to the latest update of the software to be installed within the device. The Issuer of this Credentials will be the device software vendor. The Vehicle will verify the Credentials proof by querying the Ledger and then check the Integrity of the code that is sent to it by following a procedure similar to the verification of digital signatures.

In case that software code Integrity verification fails, it means that there was a tampering of the code or the code does not belong to the Pre-market Software Vendor.

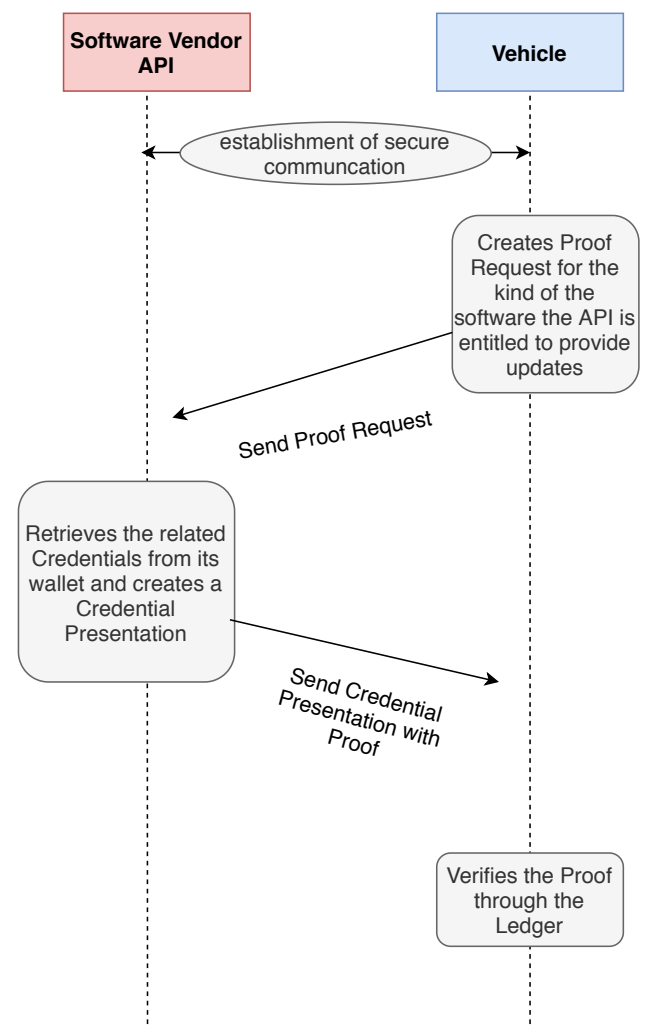

Figure 1. Authentication of the Software Vendor API by the Vehicle

The Vehicle will not install the software update. Then, a secure communication between the Vehicle and the Software Vendor will be initiated so that the Credentials of the API are revocated. ${ }^{3}$. The role of the Post-market device software vendor API is similar to the role of the Pre-market device software vendor API.

\section{CONCLUSiON \& FUture WORK}

Our choice of a DLT framework for the IdM in the IoV selects Hyperledger Indy due to the availability of advanced IdM concepts of SSI, framework expandability, and privacy preserving techniques. With the IdM capabilities of Indy, it is possible to design DID issuance and management among infrastructure stakeholders of the IoV. For stakeholder identification, the secure software update scenario between a vehicle and a cloud api defines specific ecosystem roles. Additionally, standardization formalities of cars introduce required authorities for a legitimate IdM design. The incorporation of all identities to the DID credential design establishes DLT-based credential connections of identities that are bound to Public Key Infrastructure (PKI) keypairs. Mutual authentication of credentials and connected PKI keypairs enables secure communication of software updates.

\footnotetext{
${ }^{3}$ Note that in case that the tampering of the code is done in-vehicle, we need to devise a different revocation strategy as it is mentioned in the future work section
} 
The scenario of software integrity checking as a mechanism to provoke revocation covers maintenance procedures of IdM.

Future work remains in the form of bootstrapping DIDs of Trust Anchor and Steward roles and to correct adjustments that emerge during the implementation. Additionally, the ledger requires a consortium controlled design of input verification of transactions. Addressing Indy plugins indicates the potential to enhance transaction verification of the ledger. Another issue that needs to be solved in the future is the acceptance of a revocation transaction. The reason therefore is the case of a vehicle which detects compromised software, but cannot verify whether the api or the vehicle itself caused the derogation. As a result, the decision of the Trust Anchor who submits the revocation transaction requires an extended revocation design.

\section{ACKNOWLEDGMENT}

Authors acknowledge support from the European Union's Horizon 2020 innovation action programme under grant agreement No 833742 (nIoVe).

With the support of the Technische Universität München Institute for Advanced Study, funded by the German Excellence Initiative and the European Union Seventh Framework Programme under grant agreement No 291763.

\section{REFERENCES}

[1] "Decentralized Identifiers (DIDs) v1.0," https://www.w3.org/TR/did-core/, 2020, [Online; accessed 28-February-2020].

[2] "Verifiable Credentials Data Model 1.0," https://www.w3.org/TR/vc-data-model/, 2019, [Online; accessed 28-February-2020].

[3] F. Masood and A. R. Faridi, "Distributed ledger technology for closed environment," in 2019 6th International Conference on Computing for Sustainable Global Development (INDIACom). IEEE, 2019, pp. 1151-1156.

[4] K. Li, H. Li, H. Hou, K. Li, and Y. Chen, "Proof of vote: A high-performance consensus protocol based on vote mechanism \& consortium blockchain," in 2017 IEEE 19th International Conference on High Performance Computing and Communications; IEEE 15th International Conference on Smart City; IEEE 3rd International Conference on Data Science and Systems (HPCC/SmartCity/DSS). IEEE, 2017, pp. $466-473$.

[5] S. Y. Lim, P. T. Fotsing, A. Almasri, O. Musa, M. L. M. Kiah, T. F. Ang, and R. Ismail, "Blockchain technology the identity management and authentication service disruptor: a survey," International Journal on Advanced Science, Engineering and Information Technology, vol. 8, no. 4-2, p. 1735,2018

[6] J. Kang, Z. Xiong, D. Niyato, D. Ye, D. I. Kim, and J. Zhao, "Toward secure blockchain-enabled internet of vehicles: Optimizing consensus management using reputation and contract theory," IEEE Transactions on Vehicular Technology, vol. 68, no. 3, pp. 2906-2920, 2019.
[7] B. Curran and J. Richards, "What are oracles," Smart Contracts, Chainlink \& "The Oracle Problem". Accessed, vol. $15,2019$.

[8] A. Kosba, A. Miller, E. Shi, Z. Wen, and C. Papamanthou, "Hawk: The blockchain model of cryptography and privacy-preserving smart contracts," in 2016 IEEE symposium on security and privacy (SP). IEEE, 2016, pp. 839-858.

[9] S. Kim, Y. Kwon, and S. Cho, "A survey of scalability solutions on blockchain," in 2018 International Conference on Information and Communication Technology Convergence (ICTC). IEEE, 2018, pp. 1204-1207.

[10] J. Prieto, A. K. Das, S. Ferretti, A. Pinto, and J. M. Corchado, Blockchain and Applications: International Congress. Springer, 2019, vol. 1010.

[11] M. Baza, M. Nabil, N. Lasla, K. Fidan, M. Mahmoud, and M. Abdallah, "Blockchain-based firmware update scheme tailored for autonomous vehicles," 2019 IEEE Wireless Communications and Networking Conference (WCNC), Marrakesh, Morocco, pp. 1-7, 2019.

[12] B. Lee and J.-H. Lee, "Blockchain-based secure firmware update for embedded devices in an internet of things environment," The Journal of Supercomputing, vol. 73, no. 3, pp. 1152-1167, 2017.

[13] A. Yohan and N.-W. Lo, "An over-the-blockchain firmware update framework for iot devices," 2018 IEEE Conference on Dependable and Secure Computing (DSC), Kaohsiung, Taiwan, pp. 1-8, 2018.

[14] G. Wood, "Ethereum: A secure decentralised generalised transaction ledger."

[15] “Ockam,” https://www.ockam.io/, 2017, [Online; accessed 05-March-2020].

[16] K. Olson, M. Bowman, J. Mitchell, S. Amundson, D. Middleton, and C. Montgomery, "Sawtooth: An introduction," The Linux Foundation, Jan, 2018.

[17] T. L. Foundation, "Hyperledger Indy Project," https://www.hyperledger.org/projects/hyperledger-indy, [Online; accessed 27-February-2020].

[18] C. Cachin et al., "Architecture of the hyperledger blockchain fabric," in Workshop on distributed cryptocurrencies and consensus ledgers, vol. 310, 2016, p. 4.

[19] "Car registration documents and formalities," https://europa.eu/youreurope/citizens/vehicles/registration/ formalities/index_en.htm, 2019, [Online; accessed 05-March-2020].

[20] "Exploring Hyperledger Indy through indy-dev Example," https://medium.com/@ kctheservant/exploring-hyperledgerindy-through-indy-dev-example-10075d2547/, 2019, [Online; accessed 14-March-2020].

[21] "Indy Walkthrough," https://github.com/hyperledger/indysdk/blob/master/docs/getting-started/indy-walkthrough.md, 2020, [Online; accessed 14-March-2020]. 